\title{
Mapeamento comportamental: observação de crianças no parque da pré-escola ${ }^{1}$
}

\author{
Luana dos Santos Raymundo \\ Ariane Kuhnen ${ }^{2}$ \\ Lia Brioschi Soares \\ Universidade Federal de Santa Catarina, Florianópolis-SC, Brasil
}

\begin{abstract}
Resumo: O presente artigo tem por objetivo descrever a aplicação do mapeamento comportamental centrado no lugar no contexto de um parque escolar. Esta técnica característica da psicologia ambiental relaciona o comportamento investigado com o local onde ele ocorre. Participaram do estudo cerca de 65 crianças de 3 a 5 anos de uma instituição escolar. O planejamento envolveu a definição de 11 categorias comportamentais. Este artigo constitui um recorte dos resultados evidenciando a preferência por determinados setores do espaço pelas crianças. Discutimos a importância dos estudos observacionais de fácil codificação para serem vertidos em intervenções práticas na realidade pesquisada.
\end{abstract}

Palavras-chave: psicologia ambiental, métodos de observação, comportamento de brincar.

\section{Behavioral mapping: observation of children in a preschool playground}

\begin{abstract}
This paper describes the application of behavioral mapping centered on the space of a preschool playground. This technique, characteristic of Environmental Psychology, relates the studied behavior to the place where it occurs. A total of 65 children, aged 3 to 5 years old, from a school participated in the study. The planning process involved the definition of 11 behavioral categories. This paper presents part of the results and reveals that children had a preference for certain areas of the space. We discuss the importance of observational studies with easy coding to be implemented in practical interventions in the studied context.
\end{abstract}

Keywords: environmental psychology, observation methods, childhood play behavior.

\section{Cartografía de comportamiento: la observación del niño en el parque pre-escolar}

\begin{abstract}
Resumen: Este trabajo tiene como objetivo describir la aplicación de la asignación de espacio de comportamiento centrado en el parque de una escuela. Esta característica técnica de la psicología ambiental relata el comportamiento investigado con el lugar donde se produce. En el estudio participaron cerca de 65 niños de 3 a 5 años en una escuela. La planificación implicaba la creación de 11 categorías de comportamiento. Este texto sólo proporciona un recorte de los resultados, que muestran la preferencia de los niños por ciertos sectores del espacio. Se discute la importancia de los estudios observacionales, de fácil codificación, para se remitir en intervenciones prácticas en la realidad estudiada.
\end{abstract}

Palabras clave: psicología ambiental, métodos de observación, conducta de jugar.

A psicologia ambiental contém uma abordagem interdisciplinar pela qual realiza um diálogo metodológico, por exemplo, com a arquitetura e a pedagogia, ao discutir a relação entre os aspectos físicos do ambiente e o projeto político-pedagógico da escola. O ambiente físico representa uma área fundamental para investigação na psicologia ambiental, pois é um importante aspecto da realidade, que se impinge sobre os seres humanos, e que colabora na organização de suas cognições, sentimentos e ações (Evans, 2005).

As implicações metodológicas da relação pessoaambiente exigem que a psicologia ambiental se utilize de multimétodos (Günther, Elali, \& Pinheiro, 2004). Neles a estratégia de campo, por exemplo, pode aliar a observação

\footnotetext{
${ }^{1}$ Este texto foi revisado seguindo o Acordo Ortográfico da Língua Portuguesa (1990), em vigor a partir de $1^{\circ}$ de janeiro de 2009.

${ }^{2}$ Endereço para correspondência:

Ariane Kuhnen. Universidade Federal de Santa Catarina, campus universitário Trindade. Laboratório de Psicologia Ambiental. CEP 88.040900. Florianópolis-SC, Brasil.E-mail: ariane@cfh.ufsc.br
}

com a entrevista dos participantes considerando-os como componentes do seu meio, o que facilita a validade ecológica do trabalho, conceito que se refere à possibilidade da pesquisa refletir a natureza do ambiente em que está baseada ou inserida (Günther e cols., 2004).

Os estudos direcionados à relação criança-ambiente acrescentam a esse conhecimento científico evidências de que há um forte efeito do contexto ambiental sobre o desenvolvimento infantil (Campos-de-Carvalho \& Souza, 2008). $\mathrm{O}$ contexto no qual as crianças brincam relaciona-se com a maneira como elas interagem entre si e é promotor de qualidade de vida e saúde (Fernandes \& Elali, 2008; Sager, Sperb, Roazzi, \& Martins, 2003).

Enquanto contexto de desenvolvimento infantil, o ambiente escolar atualmente apresenta uma estrutura de lugares bem diferenciados (locais para atividades livres, descanso, locais e tempo para comer etc.), ou seja, o espaço escolar possui uma grande diferença ecológica interna que interfere na relação estabelecida entre a criança e o ambiente. Entretanto, a organização do espaço na educação infantil enquanto elemento de aprendizagem, visando atender funções 
relativas ao desenvolvimento humano, ainda é novidade entre os profissionais que ali atuam (Zamberlan, Basani, \& Araldi, 2007). Desconhece-se que o desenvolvimento de capacidades cognitivas e sociais, como variável dependente, pode ser medido através da socialização e do comportamento exploratório das crianças nos espaços físicos, pois o desenho arquitetônico de cada lugar dentro da escola (variável independente) é potencializador dessas habilidades (Gilmartín, 2002).

Assim, os parques na educação infantil são lugares de aprendizagem privilegiados. Além de oportunizarem os mais variados movimentos corporais da criança - já que é importante que ela ande, corra, suba e desça, balance, pendure-se, empurre e puxe objetos - eles estimulam os sentidos. Dessa forma, quanto mais naturais forem esses espaços, maior diversidade de estímulos sensitivos conterão (Arbogast, Kane, Kirwan, \& Hertel, 2009). O tipo do solo, a incidência solar e a diversidade de cores são atrativos para as crianças quando proporcionam também conforto e segurança, ou seja, quando possibilitam que ela aventurese no espaço (dito) perigoso sem se machucar (Staempfli, 2008).

Ao tentar investigar a problemática da relevância espacial para o desenvolvimento infantil, buscando conhecer quais aspectos do espaço físico do parque são mediadores nas interações sociais e atividades das crianças, este artigo tem por objetivo apresentar uma parte da metodologia observacional aplicada na investigação, bem como um recorte dos resultados obtidos. O estudo buscou auxiliar a instituição pesquisada quanto ao uso e a ocupação de seus espaços abertos, oferecendo-lhe subsídios e diretrizes para a reorganização destes, a fim de que se tornem lugares promotores de desenvolvimento, bem-estar e saúde.

\section{Método}

Segundo Danna e Matos (2006), o método da observação se caracteriza por ser sistemático e objetivo; nesse sentido, deve ser planejado e conduzido em função de um objetivo anteriormente definido. Para isso é necessário estabelecer onde, quando e como será a observação; e quem ou o que será observado. A veracidade das representações encontradas nas pesquisas pode ser obtida através de procedimentos metodológicos básicos como a definição dos procedimentos de coleta de dados, a forma do registro e do protocolo de observação, a definição das categorias e a realização do teste de concordância. Embasada nesses critérios, a aplicação da técnica do mapeamento comportamental centrado no lugar orienta a sistematização da coleta de dados por meio de um documento científico (diagrama ou mapa) que representa graficamente as localizações e os comportamentos das pessoas no espaço. Esse mapa relaciona o espaço físico, delimitado e subdividido, e o comportamento dos usuários, classificado em categorias definidas de acordo com o objetivo do estudo, oportunizando análises críticas das atividades, além da comparação com atividades que foram planejadas para o local (Pinheiro, Elali, \& Fernandes, 2008; Sommer \& Sommer, 1997).

\section{Participantes e local}

O estudo foi realizado em um espaço de educação infantil, identificado pela Secretaria Municipal de Educação (SME) de um município brasileiro. Atendia a 110 crianças de 0 a 6 anos incompletos. Possuía uma área física construída de 391,52 $\mathrm{m}^{2}$, locados em 1.140,83 $\mathrm{m}^{2}$ de terreno, sendo a área aberta dividida em dois ambientes principais: miniquadra, com piso de concreto e $160 \mathrm{~m}^{2}$, e parque, com cobertura total do solo por areia, presença de vários equipamentos de playground, uma árvore central de pequeno porte e um total de $266,91 \mathrm{~m}^{2}$.

Foram informantes da pesquisa, os dirigentes (diretora e orientadora pedagógica) e as professoras responsáveis pelas turmas de crianças participantes. Foram observadas as crianças de 3 a 5 anos de idade, matriculadas em período integral, pertencentes a três grupos que utilizavam o parque em atividades livres cotidianas e conjuntas (média de 65 crianças). Antes do início das observações sistemáticas, houve um período de adaptação entre pesquisadores e crianças, com duração de uma semana.

\section{Procedimento}

\section{Coleta de dados}

Para a aplicação da técnica, a primeira tarefa foi confeccionar um diagrama mostrando todos os detalhes físicos do espaço do parque. A área total foi dividida em dois espaços (primeira metade - frente do parque, segunda metade - fundos). Esses espaços foram subdivididos em 13 setores menores, delimitados pela dimensão de espaço ou pela presença de equipamentos de playground, visando possibilitar a observação do parque como um todo. Levando-se em conta a divisão do parque em dois espaços, foi necessária a presença de dois observadores em cada sessão. Enquanto um observador registrava os comportamentos ocorridos na primeira metade do parque, o outro se dedicava à segunda metade, simultaneamente. Buscou-se estabelecer uma distância-padrão das crianças observadas, considerando fatores como visibilidade e possibilidade de movimentação e escuta das mesmas.

A segunda tarefa foi listar os comportamentos a serem registrados no protocolo de observação. Com o auxílio dos profissionais entrevistados, bem como via pesquisa bibliográfica realizada, foram selecionados e listados os comportamentos que seriam registrados. Amparados pelos estudos de Cordazzo, Westphal, Tagliari, Vieira e Oliveira (2008) e Sager e cols. (2003), os comportamentos totalizaram 11 categorias, definidas sinteticamente a seguir: (a) comportamento solitário - quando havia crianças no setor observado 
que brincavam de forma independente, ficando longe das outras (mais de $1 \mathrm{~m}$ de distância); (b) interação paralela quando havia crianças exercendo atividades sem que uma interviesse na brincadeira da outra; (c) interação associativa - quando havia crianças brincando umas com as outras, conversando sobre temas comuns da atividade; nessa categoria registrava-se também a composição grupal, em forma de díade (duas crianças), tríade (três crianças) ou políade (quatro ou mais crianças); (d) brincadeira de faz de conta - quando havia uma situação imaginária criada pela criança e que poderia envolver a representação de papéis; (e) brincadeira construtiva - quando envolvia uma construção; as crianças poderiam estar manipulando qualquer tipo de objeto, desde que estivessem com a clara intenção de construir algo; (f) brincadeira de jogos de regra - caracterizada pela existência de uma atividade formada por um conjunto de regras impostas e aprovadas pelo grupo participante; (g) brincadeira proposta - quando as crianças seguiam a proposta de brincadeira que sugeriu o fabricante do objeto utilizado para brincar; (h) brincadeira turbulenta - envolvia correr, empurrar, perseguir e/ou lutar e ocorria quando havia crianças que exibiam movimentos bruscos e vigorosos, porém manifestando expressões faciais hilariantes; (i) atividade de conversa - quando havia crianças envolvidas em atividade verbal com outra pessoa sem que fosse uma atividade lúdica; (j) agressão - incluía qualquer tipo de ameaça física ou verbal, com reações que indicassem situações de desentendimentos; (k) exploração do ambiente - quando havia crianças mantendo o olhar focado em algum objeto do espaço, manipulando-o sem brincar com ele.

Os seguintes aspectos das sessões também foram registrados: horário de início e término, total de crianças no parque, número e sexo de ocupantes em cada setor observado, bem como presença ou ausência de adultos. Verificou-se que 40 minutos correspondiam ao tempo médio de permanência das crianças no parque. Constatou-se também a necessidade de cinco sessões de observação em cada espaço observado para a obtenção de uma amostra fidedigna da realidade pesquisada.

As sessões se basearam em extratos instantâneos de comportamentos. Trabalhou-se a partir da noção de instante congelado de tempo (Pinheiro e cols., 2008), ou seja, os registros representavam fotografias mentais de momentos específicos das atividades das crianças. O tipo de registro efetuado foi por amostragem de tempo, de 5 em 5 minutos, a cada início do intervalo; observava-se o setor e registrava-se a ocorrência das categorias comportamentais. Cada sessão perfez oito intervalos de tempo, compreendidos em 40 minutos de observação. Uma prancheta, protocolos impressos em papel, lápis e cronômetro foram utilizados como material para a realização desse registro.

\section{Análise dos dados}

Foi realizado um teste de concordância entre observadores, necessário para garantir a precisão ou a fidedignidade das categorias comportamentais observadas e registradas. No mínimo, $70 \%$ de acordo entre os observadores é exigido ao se usar um conjunto de categorias prédefinidas (Fagundes, 1999). Para tanto, a mesma área do parque foi observada em três sessões, na mesma hora, mas de forma independente pelos dois observadores. Posteriormente, os registros foram comparados, a fim de se verificar em que medida havia concordância entre eles. Obteve-se, nas três sessões, índice de concordância superior a $85 \%$. O tratamento dos dados coletados envolveu análise estatística descritiva, que contou com o auxílio do pacote estatístico Statistical Package for Social Sciences (SPSS).

\section{Considerações éticas}

A autorização para a realização da pesquisa foi concedida pela direção da instituição e pelo Departamento de Educação Infantil da Secretaria Municipal de Educação do município. Como primeiro passo ético do estudo, o projeto foi submetido à apreciação do Comitê de Ética em Pesquisa com Seres Humanos da Universidade Federal de Santa Catarina, de acordo com as normas da Resolução no 196/196 do Conselho Nacional de Saúde. Dessa forma, foi apresentado o Termo de Consentimento Livre e Esclarecido aos responsáveis pelos participantes da pesquisa e aos profissionais da instituição, que autorizaram a participação dos infantes, via sua assinatura, garantindo-se o anonimato das pessoas envolvidas.

\section{Resultados e Discussão}

Como resultado preliminar do estudo, obteve-se uma série de mapas a intervalos representativos ao longo do período de recreação no parque: 520 mapas, somando-se as cinco sessões realizadas para cada setor. Os mapas individuais foram combinados em um único mapa composto, mostrando a ocupação e a forma de utilização do setor, traduzida nas 11 categorias comportamentais já descritas anteriormente (Figura 1). Em cada setor do parque foi possível identificar o predomínio de uma forma de interação social e de determinadas atividades. Neste artigo, discutiremos apenas os dados referentes à ocupação dos setores. 

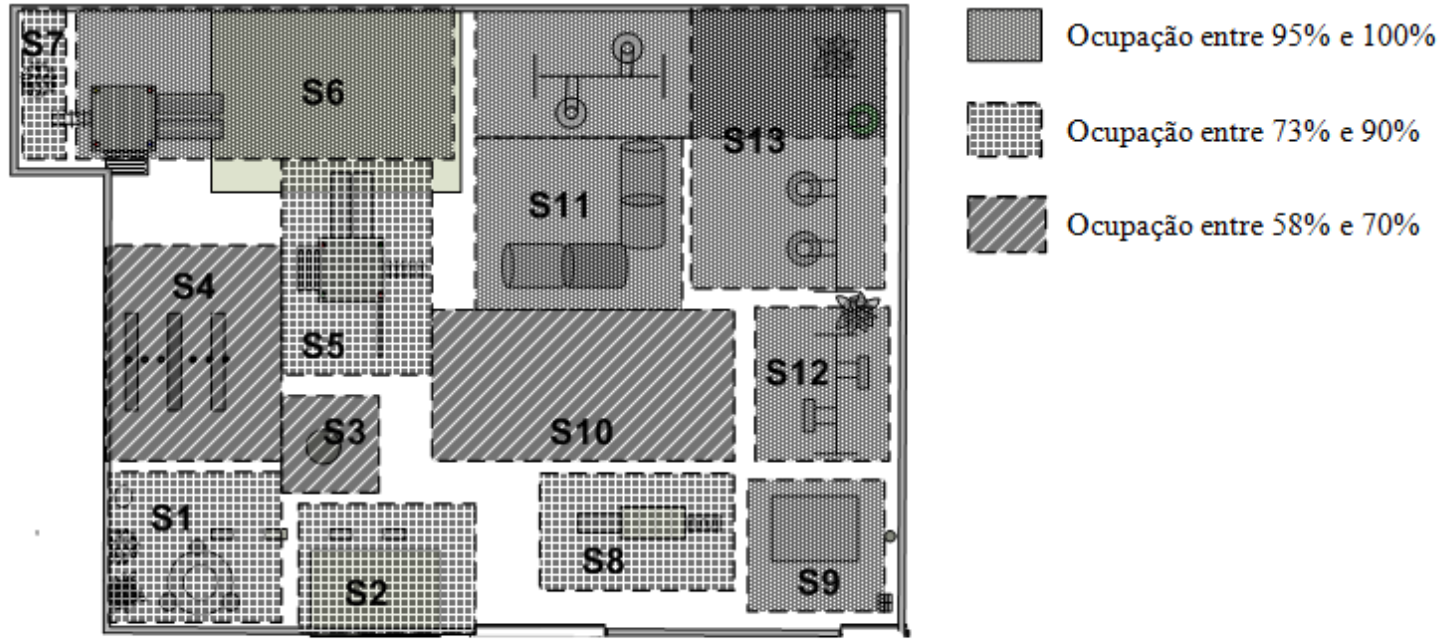

Legenda: S1 - Trepneu; S2 - Caixa de Areia; S3 - Carrossel; S4 - Gangorra; S5 - Bombeiro Duplo; S6 - Bombeiro Simples; S7 - Canto Mirante; S8 - Escorregador; S9 - Casinha de Boneca; S10 - Árvore; S11 - Túnel; S12 - Balanço Duplo; S13 - Balanço Pneus.

Figura 1. Mapa da porcentagem de ocupação dos setores do parque.

De maneira geral, a análise estatística descritiva dos registros de ocupação evidenciou que o espaço aberto do parque é, em toda a sua totalidade, bastante ocupado pelas crianças. Todos os setores observados permaneceram ocupados em mais de $50 \%$ dos registros. Com isso, pudemos apresentar aos profissionais da escola os resultados indicativos da preferência por determinados equipamentos e espaços do parque pelas crianças. Ao mesmo tempo, problematizamos que a densidade populacional de $4,10 \mathrm{~m}^{2} / \mathrm{cr}$ (metro quadrado por criança) poderia estar impossibilitando o uso de um determinado equipamento pelos menores, fazendo com que estes ocupem os setores menos disputados, como a gangorra (S4), o carrossel (S3) e a árvore (S10) (Figura 1). Ou seja, devemos considerar que a qualidade dos ambientes depende da diversidade de atividades e estímulos convidativos para o brincar, da flexibilidade ao rearranjo e da inclusão de diferentes faixas etárias nesses espaços (Campos-de-Carvalho \& Souza, 2008).

As meninas foram as responsáveis pela maior frequência de ocupação dos equipamentos de balanço e da caixa de areia, e os meninos foram os principais usuários dos equipamentos multifuncionais. Isso nos indica, conforme estudo de Cordazzo e cols. (2008), que a brincadeira é um fenômeno sensível às condições contextuais (físicas) do lugar em que ocorre, mas ao mesmo tempo, está sob controle do próprio organismo (idade e gênero). Com relação aos tipos de brincadeiras, a atividade proposta pelo equipamento (como escorregar, balançar e escalar) revestiu-se do faz de conta, principalmente nos equipamentos mais multifuncionais como a casinha de bonecas, a caixa de areia e os equipamentos de bombeiro. Esse resultado corrobora os estudos que mostram que, quanto mais flexíveis forem os espaços, maior diversidade de uso eles promoverão (Gilmartín, 2002; Sager e cols., 2003).
Por esse motivo, fez-se necessário caracterizar a ocupação do parque e discutir também a qualidade dos espaços, observando se, de fato, são mediadores de brincadeiras e possibilitam interações sociais entre as crianças. Assim, sugerimos que as instituições de educação possam conhecer tanto estudos observacionais como suas ferramentas metodológicas e deles usufruir.

\section{Considerações finais}

A técnica do mapeamento comportamental centrado no lugar permitiu a identificação de comportamentos e contatos sociais que acrescentam experiências e aprendizagens na vida das crianças. Além disso, trouxe para a instituição diretrizes para um projeto político-pedagógico que contemple o espaço físico escolar. Os resultados permitem planejar um constante rearranjo do parque, levando em consideração não só as preferências das crianças, mas também as suas necessidades desenvolvimentais.

\section{Referências}

Arbogast, K. L., Kane, B. C. P., Kirwan, J. L., \& Hertel, B. R. (2009). Vegetation and outdoor recess time at elementary schools: What are the connections? [Versão eletrônica]. Journal of Environmental Psychology, 29(4), 450-456. Recuperado em 14 agosto 2009, de http://www.sciencedirect.com/science/article/pii/ S0272494409000255

Campos-de-Carvalho, M., \& Souza, T. N. (2008). Psicologia ambiental, psicologia do desenvolvimento e educação infantil: Integração possível? Paidéia (Ribeirão Preto), 18(39), 25-40. 
Cordazzo, S. T. D., Westphal, J. P., Tagliari, F. B., Vieira, M. L., \& Oliveira, A. M. F. (2008). Metodologia observacional para o estudo do brincar na escola. Avaliação Psicológica, 7(3), 427-438.

Danna, M. F., \& Matos, M. A. (2006). Aprendendo a observar. São Paulo: EDICON.

Evans, G. (2005). A importância do ambiente físico. Psicologia USP, 16(1/2), 47-52.

Fagundes, A. J. F. M. (1999). Descrição, definição e registro de comportamento (12a ed.). São Paulo: EDICON.

Fernandes, O. S., \& Elali, G. A. (2008). Reflexões sobre o comportamento infantil em um pátio escolar: $\mathrm{O}$ que aprendemos observando as atividades das crianças. Paidéia (Ribeirão Preto), 18(39), 41-52.

Gilmartín, M. A. (2002). Ambientes escolares. In J. I. Aragonês \& M. Américo (Eds.), Psicología ambiental (pp. 221-237). Madrid: Pirâmide.

Günther, H., Elali, G. A., \& Pinheiro, J. Q. (2004). A abordagem multimétodos em estudos pessoa-ambiente: Características, definições e implicações [Versão eletrônica]. Brasília: Laboratório de Psicologia Ambiental - Universidade de Brasília. (Série: Textos de Psicologia Ambiental, 23). Recuperado em 06 junho 2008, de http:// www.psi-ambiental.net/XTextos/20MultiMetodo.pdf

Pinheiro, J. Q., Elali, G. A., \& Fernandes, O. S. (2008). Observando a interação pessoa-ambiente: Vestígios ambientais e mapeamento comportamental. In J. Q. Pinheiro \& H. Günther (Orgs.), Métodos de pesquisa nos estudos pessoa-ambiente (pp. 75-104). São Paulo: Casa do Psicólogo.

Sager, F., Sperb, T. M., Roazzi, A., \& Martins, F. M. (2003). Avaliação da interação de crianças em pátios de escolas infantis: Uma abordagem da psicologia ambiental. Psicologia: Reflexão e Crítica, 16(1), 203-215.

Sommer, R., \& Sommer, B. B. (1997). Behavioral mapping. In R. Sommer \& B. B. Sommer, A practical guide to behavioral research: Tools and techniques (3rd ed., pp. 60-78). New York: Oxford University Press.

Staempfli, M. (2009). Reintroducing adventure into children's outdoor play environments [Versão Eletrônica]. Environment and Behavior, 41(2), 268-280. Recuperado em 14 agosto 2009, de http://eab.sagepub. com/content/41/2/268.full.pdf

Zamberlan, M. A. T., Basani, S. I. S., \& Araldi, M. (2007). Organização do espaço e qualidade de vida: Pesquisa sobre configuração espacial em uma instituição de educação infantil. Educere et Educare, 2(4), 245-260.
Luana dos Santos Raymundo é doutoranda em Psicologia pelo Programa de Pós-graduação em Psicologia da Universidade Federal de Santa Catarina.

Ariane Kuhnen é Professora Doutora do Programa de Pósgraduação em Psicologia da Universidade Federal de Santa Catarina .

Lia Brioschi Soares é psicóloga graduada pela Universidade Federal de Santa Catarina.
Recebido: 28/08/2010

$1^{a}$ revisão: $23 / 10 / 2010$

$2^{a}$ revisão: $23 / 02 / 2011$

Aceite final: 01/03/2011 